\title{
Article \\ Genetic Dominant Variants in STUB1, Segregating in Families with SCA48, Display In Vitro Functional Impairments Indistinctive from Recessive Variants Associated with SCAR16
}

\author{
Yasaman Pakdaman ${ }^{1,2}$, Siren Berland ${ }^{1}$, Helene J. Bustad ${ }^{3}{ }^{(D}$, Sigrid Erdal ${ }^{1}$, Bryony A. Thompson ${ }^{4,5}$, \\ Paul A. James ${ }^{6,7}$, Kjersti N. Power ${ }^{8}$, Ståle Ellingsen ${ }^{9}$, Martin Krooni ${ }^{10,11}$, Line I. Berge ${ }^{10,12}$, Adrienne Sexton ${ }^{6,7}$, \\ Laurence A. Bindoff ${ }^{13,14}$, Per M. Knappskog ${ }^{1,2}$, Stefan Johansson ${ }^{1,2}$ and Ingvild Aukrust ${ }^{1,2, *}$
}

check for updates

Citation: Pakdaman, Y.; Berland, S.; Bustad, H.J.; Erdal, S.; Thompson, B.A.; James, P.A.; Power, K.N.; Ellingsen, S.; Krooni, M.; Berge, L.I.; et al. Genetic Dominant Variants in STUB1, Segregating in Families with SCA48, Display In Vitro Functional Impairments Indistinctive from Recessive Variants Associated with SCAR16. Int. J. Mol. Sci. 2021, 22, 5870. https://doi.org/10.3390/ ijms22115870

Academic Editor: Ginevra Zann

Received: 20 April 2021

Accepted: 26 May 2021

Published: 30 May 2021

Publisher's Note: MDPI stays neutral with regard to jurisdictional claims in published maps and institutional affiliations.

Copyright: (C) 2021 by the authors Licensee MDPI, Basel, Switzerland. This article is an open access article distributed under the terms and conditions of the Creative Commons Attribution (CC BY) license (https:// creativecommons.org/licenses/by/ $4.0 /)$
1 Department of Medical Genetics, Haukeland University Hospital, 5021 Bergen, Norway; yasaman.pakdaman@uib.no (Y.P.); siren.berland@helse-bergen.no (S.B.); sigrid.erdal@helse-bergen.no (S.E.); per.morten.knappskog@helse-bergen.no (P.M.K.); Stefan.Johansson@uib.no (S.J.)

2 Department of Clinical Science, University of Bergen, 5021 Bergen, Norway

3 Department of Biomedicine, University of Bergen, 5021 Bergen, Norway;

helene.bustad.johannessen@gmail.com

4 Department of Pathology, Royal Melbourne Hospital, Parkville, VIC 3050, Australia; Bryony.Thompson@mh.org.au

5 Department of Clinical Pathology, Melbourne Medical School, University of Melbourne, Parkville, VIC 3010, Australia

6 Genomic Medicine, Royal Melbourne Hospital, Parkville, VIC 3050, Australia; Paul.James@petermac.org (P.A.J.); adrienne.sexton@mh.org.au (A.S.)

7 Department of Medicine, University of Melbourne, Parkville, VIC 3051, Australia

8 Department of Neurology, Haukeland University Hospital, 5021 Bergen, Norway; kjersti.nesheim.power@helse-bergen.no

9 Department of Biological Sciences, University of Bergen, 5006 Bergen, Norway; Stale.Ellingsen@uib.no

10 NKS Olaviken Gerontopsychiatric Hospital, 5306 Askøy, Norway; martin.krooni@gotland.se (M.K.); Line.Berge@uib.no (L.I.B.)

11 Department of Health and Medical Care, Clinic of Psychiatry, Visby Hospital, 62155 Visby, Sweden

12 Centre for Elderly and Nursing Home Medicine, Department of Global Public Health and Primary Care, University of Bergen, 5009 Bergen, Norway

13 Neuro-SysMed, Center of Excellence for Clinical Research in Neurological Diseases, Department of Neurology, Haukeland University Hospital, 5021 Bergen, Norway; laurence.albert.bindoff@helse-bergen.no

14 Department of Clinical Medicine, University of Bergen, 5021 Bergen, Norway

* Correspondence: ingvild.aukrust@helse-bergen.no; Tel.: +47-9883-6907

Abstract: Variants in STUB1 cause both autosomal recessive (SCAR16) and dominant (SCA48) spinocerebellar ataxia. Reports from 18 STUB1 variants causing SCA48 show that the clinical picture includes later-onset ataxia with a cerebellar cognitive affective syndrome and varying clinical overlap with SCAR16. However, little is known about the molecular properties of dominant STUB1 variants. Here, we describe three SCA48 families with novel, dominantly inherited STUB1 variants (p.Arg51_Ile53delinsProAla, p.Lys143_Trp147del, and p.Gly249Val). All the patients developed symptoms from 30 years of age or later, all had cerebellar atrophy, and 4 had cognitive/psychiatric phenotypes. Investigation of the structural and functional consequences of the recombinant Cterminus of HSC70-interacting protein (CHIP) variants was performed in vitro using ubiquitin ligase activity assay, circular dichroism assay and native polyacrylamide gel electrophoresis. These studies revealed that dominantly and recessively inherited STUB1 variants showed similar biochemical defects, including impaired ubiquitin ligase activity and altered oligomerization properties of the CHIP. Our findings expand the molecular understanding of SCA48 but also mean that assumptions concerning unaffected carriers of recessive STUB1 variants in SCAR16 families must be re-evaluated. More investigations are needed to verify the disease status of SCAR16 heterozygotes and elucidate the molecular relationship between SCA48 and SCAR16 diseases.

Keywords: spinocerebellar ataxia; SCA48; SCAR16; CHIP; STUB1; E3 ubiquitin ligase 


\section{Introduction}

Dominantly inherited spinocerebellar ataxias (SCAs) are a heterogeneous group of neurodegenerative disorders characterized by adult-onset progressive cerebellar ataxia. Many also have additional neurological symptoms including pyramidal and extrapyramidal features, and cognitive decline [1]. The global prevalence of SCA is estimated to be $1-5: 10^{5}$, with the highest rates reported by population-based surveys in Portugal $\left(5.6: 10^{5}\right)$, Japan $\left(5: 10^{5}\right)$, and Southeast Norway $\left(4.2: 10^{5}\right)[2,3]$.

Expanded polyglutamine repeats (CAG; polyQ) are responsible for the most common types of SCAs, followed by other noncoding repeat expansions and substitutions [1]. Recently, heterozygous pathogenic variants (mutations) in the STUB1 gene (STIP1 homology and U-box containing protein 1; OMIM, 607207) causing a new form of SCA (SCA48) were described. The first reported Spanish family had a cognitive-affective syndrome (CCAS) and late-onset SCA following an autosomal dominant inheritance pattern [4]. Subsequent studies reported heterozygous STUB1 mutations in several families with ataxia and cognitive-psychiatric disorder, often associated with other features such as dystonia, parkinsonism, chorea, and endocrine dysfunction [5-7]. To date, 18 heterozygous pathogenic STUB1 variants have been reported to be associated with a SCA48 phenotype; however, how these mutations affect protein structure and the function remains unclear. Functional data are essential to elucidate mechanisms underlying the dominant inheritance of this disease $[8,9]$.

The C-terminus of HSC70-interacting protein (CHIP) is a co-chaperone of E3 ubiquitin ligase encoded by the STUB1 gene and acts as a component of the protein quality control system, targeting misfolded and damaged proteins for ubiquitination and subsequent degradation through proteasome and/or autophagy pathways [10]. The active CHIP functions as a dimeric protein consisting of an $\mathrm{N}$-terminal tetratricopeptide repeat (TPR) domain (residues 26-127) mainly involved in the interaction of the CHIP with molecular chaperones, and a C-terminal U-box domain (residues 226-297) which functions as an E3 ubiquitin ligase. The two domains are separated by a central helical hairpin region (residues 128-225) that is critical for the stability and dimerization of the CHIP. The CHIP dimerization is also mediated by a second interacting surface in the U-box domain [11]. The CHIP is highly expressed in metabolically active tissues such as the brain and plays a protective role to prevent accumulation of abnormal proteins and neurodegeneration (10). Bi-allelic mutations in STUB1 cause autosomal recessive spinocerebellar ataxia (SCAR16), an early-onset disorder with gait disturbance, dysarthria, head and hand tremor, hyperreflexia, cognitive decline, and, occasionally, hypogonadism [12]. SCAR16 variants are thought to destabilize the CHIP structure and lead to mutation-specific abnormalities such as decreased interaction with chaperones, reduced steady-state cellular levels, reduced ubiquitination activity, and/or misfolding [13,14].

In this study, we report three novel heterozygous STUB1 variants in families presenting with SCA48 phenotypes. To understand the functional effects of these variants, we characterized the ubiquitin ligase activity and examined the structural properties of these variants using the recombinant CHIP purified from Escherichia coli. Our results suggest that the variants causing SCA48 are indistinguishable with regard to their functional impairment from previously described variants reported in families with SCAR16.

\section{Results}

\subsection{Genetic Findings}

Pedigrees of the three families with heterozygous STUB1 variants segregating with dominant SCA48 are presented in Figure 1. Variants in STUB1 (NM_005861.3) were detected by exome sequencing: in Family A, the proband, her father, and two of her three siblings carried the heterozygous variant c.152_158delinsCAGC p.(Arg51_Ile53delinsProAla) (abbreviated as R51_I53delinsPA). All carriers in Family A showed clinical characteristics similar to previously reported cases of SCA48 (Table S1). The mother and one sister did not have symptoms of SCA48, and the sister was tested and did not carry the R51_I53delinsPA 
STUB1 variant. The R51_I53delinsPA variant is not present in the Genome Aggregation Database (gnomAD) or in the dbSNP database, and the three amino acids located in the TPR domain (Arg51, Ala52 and Ile53) are well conserved throughout species. In addition to next-generation sequencing (NGS)-based gene panel analysis in all three affected siblings, the most severely affected family member, the father (II-2), was also tested for trinucleotide repeat expansions in other SCA associated genes (see Materials and Methods) with normal findings. The deceased paternal grandmother could not be tested, but according to the family, she also had ataxia and dysarthria.
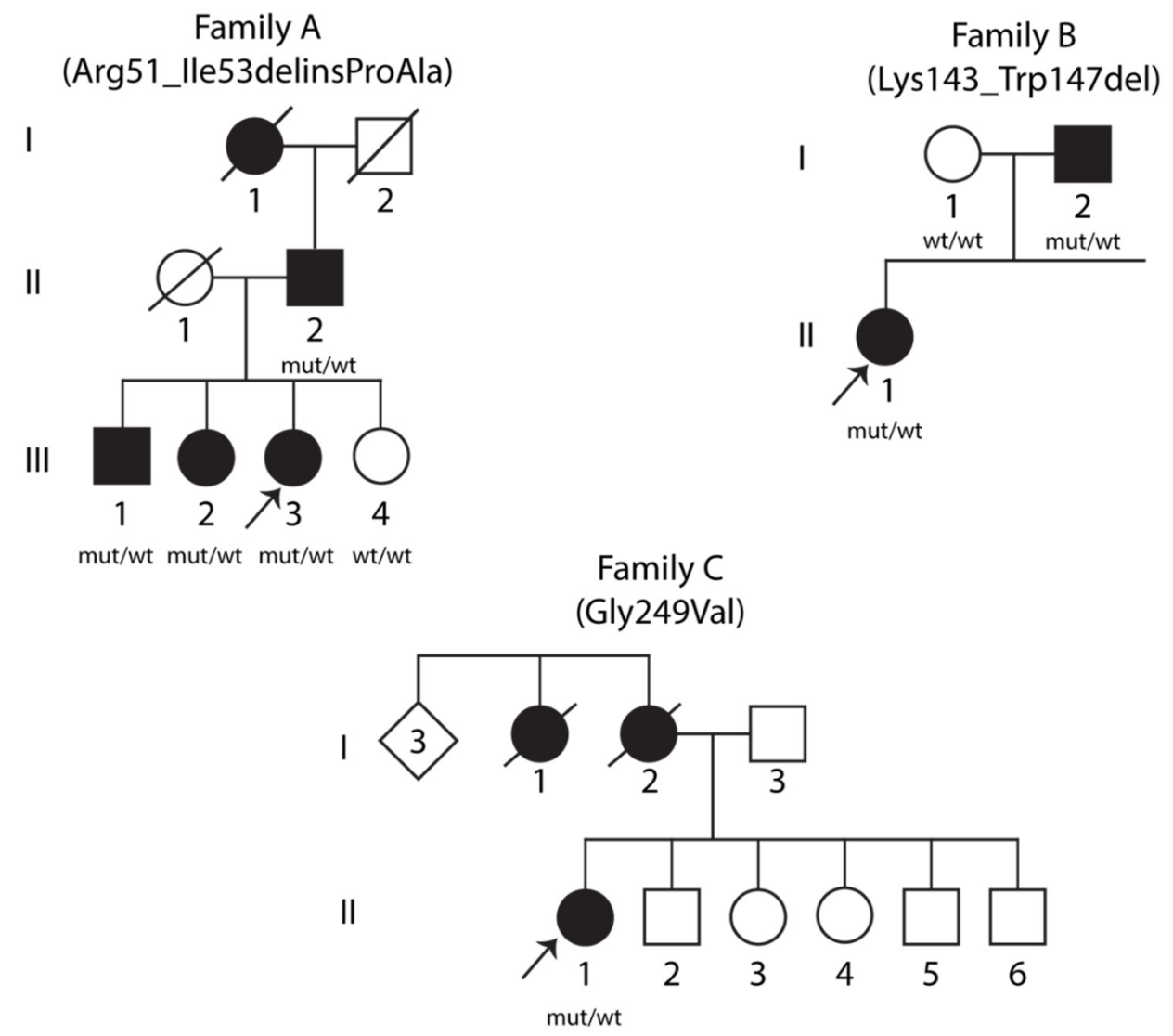

Figure 1. Pedigrees of three families with SCA48. Clinically affected individuals are indicated by filled symbols. Arrows denote the probands. Genetic statuses of examined individuals are provided below the symbols, where "mut" represents the mutant allele and " $w t^{\prime \prime}$ represents the wild-type allele.

The proband in Family B and her affected father carried the heterozygous STUB1 variant c.426_441delinsT p.(Lys143_Trp147del) (abbreviated as K143_W147del). The variant is not present in gnomAD or in the dbSNP database. The five amino acids involved (Lys143, Lys144, Lys145, Arg146 and Trp147) are located in the central helical hairpin region and are well conserved throughout species. Both the proband and her father were also heterozygous for a 41-repeat expansion in the gene coding for TATA box binding protein, TBP.

The proband in Family $\mathrm{C}$ was heterozygous for a missense variant in STUB1: c.746G $>$ T p.(Gly249Val) (abbreviated as G249V). Her mother died at age 73, not tested for the variant, and was reported to have behavioral variant frontotemporal dementia (bvFTD) from age 60. The variant is not present in gnomAD or in the dbSNP database, and Gly249, located in the U-box domain, is well conserved across species. No repeat expansions in other SCA-associated genes were found in this family. 


\subsection{Clinical Features}

Seven patients were examined from three families, with the main clinical features summarized in Table S1. The female proband in Family A (III-3) first developed dysarthria at the age of 40, and thereafter mild dysphagia. She was diagnosed with mild cerebellar gait ataxia at the age of 49. At age 51, she remains able to work part time. Her MRI showed cerebellar atrophy and four small high-signal lesions that have remained unchanged over time. Spinal fluid examination and evoked potentials were normal. The sister of the proband (III-2) developed depression at 47 years of age. Cognitive decline appeared some years later and, based on an MRI showing cerebellar atrophy, she was diagnosed with cerebellar cognitive affective syndrome (CCAS) at the age of 53 . Other clinical features appeared normal in this patient. She is not able to work. The brother of the proband (III-1) presented with dysarthria at the age of 52. Eye movement examination showed dysmetric saccades. His gait and limb coordination were normal. His MRI showed clear cerebellar atrophy. The father of the proband (II-2) was diagnosed with cerebellar ataxia at 74 years of age, having presented with gait disturbance, encephalopathy, pyramidal signs, dysarthria, and dysphagia present from the age of 50. His MRI showed marked atrophy of the cerebellum and generalized cerebral atrophy. The unaffected sister (III-4) agreed to participate in the family study. Clinical examination produced normal findings, and her MRI was normal with no signs of cerebellar atrophy.

In Family B, the female proband (II-1) was first seen for dysarthria and unsteadiness at the age of 30. Examination revealed mild cerebellar dysarthria and mild tandem gait ataxia. She subsequently progressed with increasing gait ataxia which necessitated wheelchair use, encephalopathy, and bilateral horizontal nystagmus. An MRI taken at the age of 38 showed marked global cerebellar atrophy. The father (I-2) presented with gait disturbance initially thought to be parkinsonism at the age of 69 years and was later diagnosed with cerebellar gait disturbance at the age of 74 . In addition to cerebellar atrophy, MRI findings revealed a small frontal lacunar infarct. The father is still ambulant and clinically less severely affected than his daughter.

The female proband of Family C (II-1) presented with cognitive and psychiatric symptoms associated with bvFTD disorder at the age of 48 . She was found to have an ataxic gait, peripheral cerebellar dysfunction, and dysphagia, with progressive cerebellar atrophy on her MRI. The mother died at the age of 73 and was diagnosed with bvFTD at the age of 60 . The mother's sister died of dementia in her 50s, but the mother's three siblings did not have any neurological symptoms. In Family C, only the proband was available for genetic testing.

\subsection{Hsc70- and Self-Ubiquitination Activity of the CHIP Variants}

The in vitro ability of the wild-type and variant CHIPs to ubiquitinate themselves with the Hsc70 substrate was investigated using recombinant maltose binding protein (MBP)CHIP proteins and showed impaired ubiquitination activity toward the Hsc70 substrate for all the examined variants (Figure 2A). Self-ubiquitination activity appeared intact and similar to the wild-type control for the R51_I53delinsPA and K143_W147del variants as shown by the presence of several high-molecular bands, suggesting covalent attachment of ubiquitin to the CHIP itself; the G249V variant displayed impaired self-ubiquitination activity (Figure 2B). 


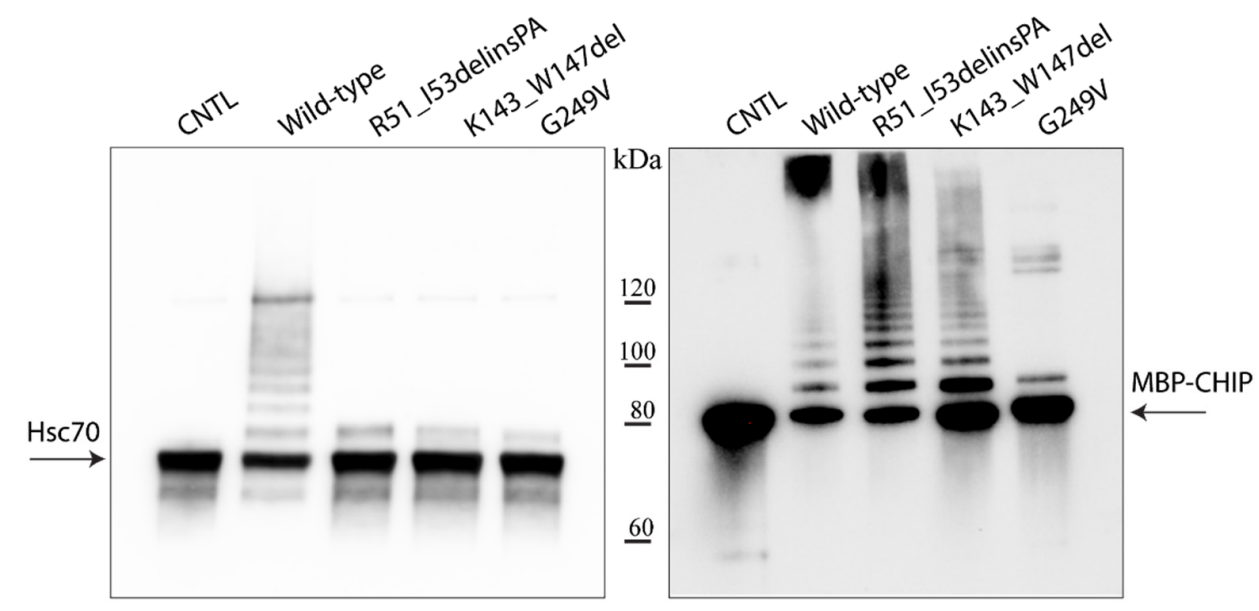

Figure 2. Ubiquitination activity of wild-type and mutant MBP-CHIP proteins. In vitro ubiquitination activity test was performed on MBP-CHIP recombinant proteins using Hsc70 (A) and $\mathrm{MBP}-\mathrm{CHIP}(\mathrm{B})$ as the substrates. A separate wild-type reaction without ubiquitin was used as the negative control (CNTL) for each assay.

\subsection{Thermal Unfolding of the CHIP Variants}

The effect of STUB1 variants on the CHIP conformational stability as a function of temperature was examined by circular dichroism spectroscopy. The changes in protein molar ellipticity were monitored by gradually increasing the temperature from $20^{\circ} \mathrm{C}$ to $90{ }^{\circ} \mathrm{C}$ (Figure 3). The thermal unfolding of the wild-type MBP-CHIP (Figure 3, blue) indicates a distinctive profile with three transitions representing denaturation of the MBP-CHIP dimers (phase 1), the MBP (phase 2), and the CHIP monomers (phase 3), as previously reported by us [13]. All three variants demonstrated a decrease in initial molar ellipticity at $222 \mathrm{~nm}$, and the loss of one or two unfolding transitions. These results indicate that the mutations have a pronounced impact both on the structure of the MBP-CHIP and on the oligomeric states, likely due to altered tertiary structures. A reduction in signal was also detected for all the variants in their far-UV circular dichroism spectra (Figure A1), supporting the loss of secondary structures. These results suggest that all three mutations induce conformational changes in their encoded CHIP proteins, resulting in the acquisition of different oligomeric states and, thus, the abnormal thermal denaturation profiles (Figure 3).

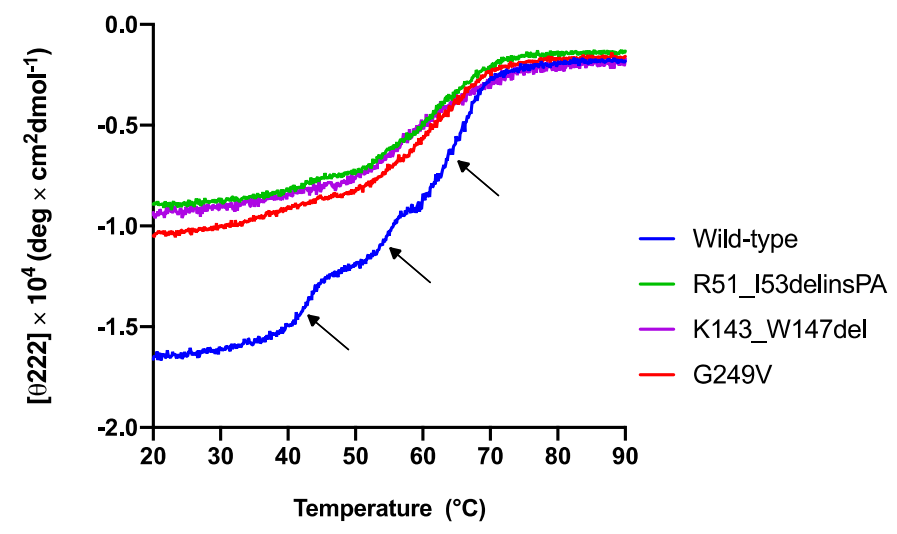

Figure 3. Thermal unfolding profiles of wild-type and mutant MBP-CHIP proteins monitored by circular dichroism spectroscopy. Thermal unfolding curves were obtained for MBP-CHIP proteins by following changes in molar ellipticity at $222 \mathrm{~nm}$ wavelength as a function of temperature. Wildtype MBP-CHIP shows three distinct transitions at approximately $42.8^{\circ} \mathrm{C}, 54.6^{\circ} \mathrm{C}$, and $65.1{ }^{\circ} \mathrm{C}$, as indicated by arrows, whereas all three variants show loss in both ellipticity and transitions. 


\subsection{Oligomerization States of the CHIP Variants}

To elucidate the effect of STUB1 variants on the native conformation and oligomerization states of the CHIP, we analyzed wild-type and mutant MBP-CHIP proteins by native polyacrylamide gel electrophoresis (native-PAGE) (Figure 4). Wild-type MBP-CHIP proteins were separated into three major bands, where the dominating band represented dimers, and the bands containing monomeric and higher order oligomeric structures appeared to a lesser extent. Examination of the mutant proteins showed a clear shift toward the higher-order oligomeric structures for all the variants, suggesting an increased propensity to form protein aggregates. While the amount of monomers remained similar for all the examined samples, dimeric structures were absent for the G249V variant, but detectable for R51_I53delinsPA and K143_W147del with 43\% and 41\% decreased intensity compared to the wild-type, respectively. Overall, these data are in accordance with the thermal unfolding profiles and indicate a profound alteration in the oligomeric structure of variants compared to the wild-type, shown by significant loss of dimers and higher-order oligomers comprising the major conformational state of mutant MBP-CHIP proteins.

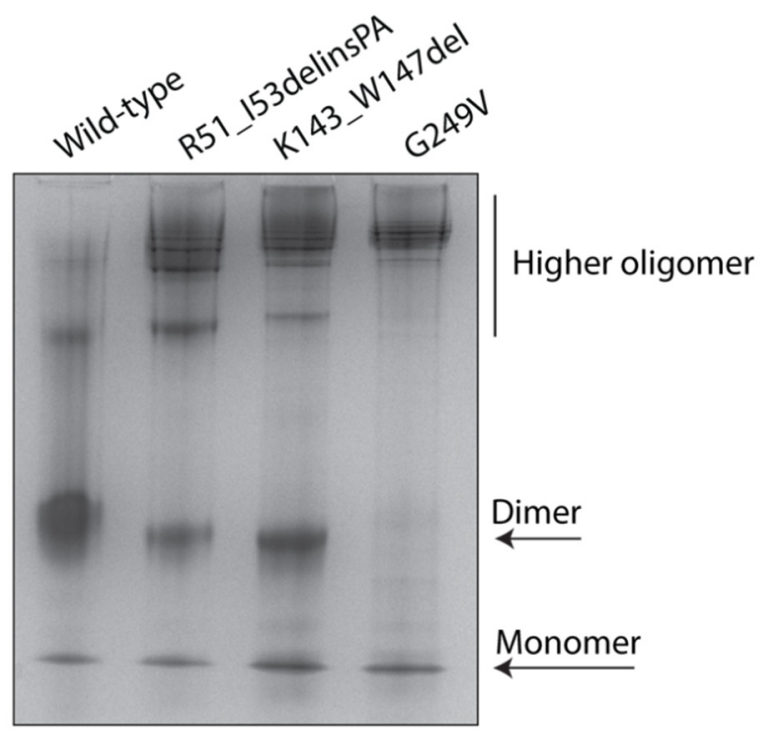

Figure 4. Oligomerization states of wild-type and mutant MBP-CHIP proteins. Native structures of $5 \mu \mathrm{g}$ MBP-CHIP proteins were studied by native-PAGE on a $10 \%$ gel. Protein bands were visualized by Coomassie blue staining and analyzed by Fiji (ImageJ) software [15].

\section{Discussion}

Heterozygous STUB1 mutations cause dominantly inherited cerebellar ataxia SCA48, and despite its recent description, SCA48 has emerged as one of the more frequent subtypes of SCA [9]. Here, we report three novel heterozygous STUB1 variants found in three families with SCA48 and describe the clinical variation associated with these variants. We also, for the first time, characterize the deleterious effect of heterozygous STUB1 mutations on the CHIP protein structure and function.

Seven patients from three families were investigated. Clinically, all patients had a phenotype compatible with that described in SCA48 [4,5,16,17], and the majority of them had an early-onset dysarthria and progressive cerebellar ataxia with cognitive impairment developing later. Parkinsonism was seen in one patient. All patients developed their first symptoms at or after the age of 30 . Despite a small sample size, we observed a tendency for females to develop a more severe phenotype, with earlier onset, than men. A sexdependent penetrance has been suggested earlier [17], but also been questioned [18], and larger cohorts are probably needed to resolve this question.

Our functional data showed impaired ubiquitination activity on the Hsc70 substrate for all the CHIP variants, while the G249V variant was also unable to perform self- 
ubiquitination. The location of these mutations in functional CHIP domains suggests that the R51_I53delinsPA variant might interfere with the CHIP's ability to target Hsc70 substrates at the TPR domain, while the G249V variant likely affects the CHIP ubiquitin ligase activity via the U-box domain, resulting in impaired ubiquitination of both the Hsc70 substrate and the CHIP itself. Further investigations into the conformational structure of the CHIP variants revealed significant alterations in protein oligomerization, demonstrated by the reduced amount of dimers and increased formation of higher-order oligomers or aggregates. Therefore, the mutations also appear to result in conformational changes that may contribute to pathogenesis in SCA48 patients through the gaining of toxic functions. This could, in particular, be the main mechanism for the K143_W147del and G249V variants, given the location of these mutations in the CHIP dimerization interfaces [19]. The dimerization of the K143_W147del variant is less severely affected, as a proportion of dimeric content is still seen. The complete lack of dimeric structures in the G249V variant, on the other hand, indicates an abolished dimerization interface in the U-box domain.

Similar defects in the protein structure and ubiquitination activity of the CHIP have also been described for bi-allelic STUB1 variants associated with the SCAR16 disease [9]. In fact, our functional data on the SCA48 variants are highly similar to what we previously reported for the SCAR16 variant, p.Thr246Met [13]. Similar to the G249V variant, this mutation is also located in the U-box domain and displays impaired ubiquitination activity and a very small number of dimers, yet a higher degree of aggregates. In addition to the functional similarities, there is a clinical overlap between the recessive and dominant disorders, where ataxia and cognitive dysfunction are common in both groups. However, an earlier age of onset and additional multi-organ involvement defines the greater severity of SCAR16. This greater severity may reflect the lack of any functional allele in the recessive disease, as suggested in studies that show more severe physiological and behavioral defects

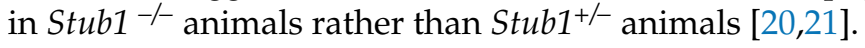

The pathogenicity of heterozygous STUB1 variants could reflect a dominant negative effect for SCA48 mutations. It remains unclear, however, why some STUB1 mutations cause recessive ataxias with apparently unaffected heterozygous parents, while similar mutations cause dominant SCA48 in other families. The SCAR16 variant p.Asn65Ser (N65S) was originally described as a cause of SCAR16 in three siblings born from healthy parents [22]. This variant was, however, was recently reported as a cause for an autosomal dominant disorder in three families, with later onset and different clinical picture compared to the original SCAR16 families [17]. Based on this, we suggest that a close follow up of STUB1 carriers in SCAR16 families is warranted. Penetrance may possibly vary to a greater degree in dominant forms, and it is probable that genetic variation elsewhere in the genome impacts the penetrance or expression of certain variants. For example, an additional variant of unknown significance in PRKCG, the gene coding for protein kinase C Gamma associated with SCA14, was seen in heterozygous carriers of N65S, suggesting a possible synergy of the two variants in the early development of disease [17]. In our Family B, both the proband and her father were heterozygous for a 41-repeat expansion in $T B P$. Repeat expansions of 41-45 in TBP have been described as intermediate penetrant alleles associated with SCA17 [23,24]; however, the pathogenicity of the 41-repeat expansion allele has been questioned since it is present at a minor allele frequency of $0.5-0.7 \%$ in control populations [25,26] and has been found in many asymptomatic individuals [27]. Since no SCA17 families with a heterozygous 41-repeat allele and dominant inheritance have been published, it is highly unlikely that this alone can cause the severe phenotype in patient II-1, and milder effects on I-2 in family B. We cannot, however, exclude a modifying effect.

In summary, our findings demonstrate similar functional and biochemical properties for the heterozygous STUB1 variants identified in this study and those of previously reported recessive variants associated with SCAR16. The reduced penetrance observed in some of the reported SCA48 families might suggest the presence of additional undiscovered genetic factors in these families. Our data also suggest that STUB1 carriers in SCAR16 families require regular clinical follow-up. Future studies are required to elucidate the 
exact genotype-phenotype correlation as well as pathways behind the pathogenesis of these variants.

\section{Materials and Methods}

\subsection{Clinical Data}

Three families with seven patients were included in this study, after having the results from diagnostic NGS gene panel analysis requested by a neurologist (Family A and B) or geneticist (Family C). The three siblings in Family A were referred by three different neurologists as separate probands, at that time not aware of the affected siblings. Clinical information regarding the patients and MRI scans is summarized in Table S1. All patients were investigated by at least one neurologist. Blood samples were collected from all available family members and DNA purified by standard methods. Written informed consent was obtained from all participants. All patients and involved families were offered genetic counselling.

\subsection{Genetic Analyses}

In Families A and B, a targeted 639 gene panel, including ataxia genes, was performed and extracted from whole exome sequencing data in III-1, III-2, and III-3 in Family A and II-1 in Family B. Whole exome sequencing was performed using genomic DNA from the probands. DNA samples were prepared using the SeqCap EZ MedExome target enrichment kit (Roche NimbleGen, Madison, WI, USA) and then underwent paired-end 150 nucleotide sequencing on the Illumina NextSeq500 (Illumina, Way San Diego, CA, USA). Alignment and variant calling were performed as previously described [28,29]. Data annotation and interpretation were performed using the Cartagenia Bench Lab, NGS module (Cartagenia, Leuven, Belgium). The STUB1 variants in Families A and B were verified by Sanger sequencing in the probands and in affected family members. The affected fathers (I-2) in both Family A and B were not analyzed by NGS, only by Sanger sequencing of the relevant STUB1 variant. In patient II-1 from Family C, exome sequencing was performed by the Australian Genome Research Facility Ltd., with the SureSelect XT Low Input Clinical Research Exome V2 (Agilent Technologies, CA, USA) kit and sequencing on an Illumina NovaSeq6000. Data were processed using the GATK best-practice pipeline v4.0 (Broad Institute, Cambridge, MA, USA), including alignment to the GRCh38 reference genome and variant calling using HaplotypeCaller. Variant prioritization using a 116 gene target panel (including adult-onset ataxia genes and early-onset dementia genes) was performed using Alissa Interpret v5.2.7 (Agilent Technologies, CA, USA). Patient II-2 in Family A, patients I-2 and II- 1 in Family B, and patient II- 1 in Family $C$ were tested for trinucleotide repeat expansions in ATXN1 (SCA1), ATXN2 (SCA2), ATXN3 (SCA3), CACNA1A (SCA6), and ATXN7 (SCA7). Further, patient II-2 in Family A and patients I-2 and II-1 in Family B were also tested for repeat expansions in PPP2R2B (SCA12), TBP (SCA17), ATN1 (DRPLA), and FXN (FRDA). Patient II-1 in Family C was also tested for expansions in HTT. FMR1 repeat expansions were excluded in all four affected patients in Family A, and in the father I-2 in Family B. In patient II- 1 in Family B, whole genome sequencing (WGS) was performed at the Genomics Core Facility (University of Bergen, Bergen, Norway) (only looking at the STUB1 gene) to search for a second STUB1 variant (deletion or deep intronic variant), with no second finding. WGS was performed as previously described [28]. Furthermore, haplotype analyses revealed that the three affected siblings in Family A did not share the same maternal STUB1 allele.

\subsection{In Vitro Expression of Mutant CHIP Proteins}

The full-length wild-type STUB1 cDNA was previously cloned into the bacterial expression vector His6-MBP-pETM-41 [13]. The plasmid was used to generate STUB1 constructs containing c.152_158delinsCAGC, c.426_441delinsT and c.746G > T by QuikChange XL Site-directed Mutagenesis Kit (Agilent Technologies) (mutagenesis primers available upon request). Wild-type and mutant MBP-CHIP fusion proteins were further expressed 
overnight at $25^{\circ} \mathrm{C}$ in BL21-CodonPlus (DE3)-RP cells (Agilent Technologies). The Histagged proteins were then purified by Ni-NTA agarose nickel affinity binding resin (QIAGEN, Hilden, Germany), following the manufacturer's instructions. The CHIP proteins were stored in $100 \mathrm{mM}$ HEPES, $100 \mathrm{mM} \mathrm{NaCl}, 5 \mathrm{mM}$ DTT, and $10 \%$ glycerol at $-80{ }^{\circ} \mathrm{C}$.

\subsection{In Vitro Ubiquitination Activity Assay}

An in vitro ubiquitination activity assay was set up for the wild-type and mutant MBP-CHIP proteins in a $30 \mu \mathrm{L}$ reaction containing $2.5 \mu \mathrm{g}$ CHIP and $1 \mu \mathrm{g}$ His-HSPA8 /HSC70 (LifeSpan BioSciences, Seattle, WA, USA) incubated with $250 \mu \mathrm{M}$ ubiquitin (Boston Biochem, MA, USA), $2.5 \mu \mathrm{M}$ UbcH5c (E2) (Boston Biochem), $0.05 \mu \mathrm{M}$ Ube1 (E1) (Boston Biochem), $50 \mathrm{mM}$ Tris HCl, $\mathrm{pH} 7.5,0.6 \mathrm{mM}$ DTT, and $2.5 \mathrm{mM} \mathrm{Mg}$-ATP for $1 \mathrm{~h}$ at $37^{\circ} \mathrm{C}$. The negative control samples were prepared using wild-type CHIP proteins and without adding ubiquitin to the reaction mixture. Samples were analyzed for CHIP and Hsc70 ubiquitination by sodium dodecyl sulfate (SDS)-PAGE and Western blotting using antiHSC70/HSP73 (1:10,000, Enzo Life Sciences ADI-SPA-815, UK) and anti-CHIP (1:2000, Sigma C9243, MO, USA) antibodies.

\subsection{Circular Dichroism Spectroscopy}

Circular dichroism far-UV and thermal denaturation measurements were recorded using a Jasco J-810 spectropolarimeter equipped with a CDF-426S Peltier temperature control unit (Jasco Products, OK, USA). The wild-type and mutant MBP-CHIP proteins were prepared at $2.5-5.8 \mu \mathrm{M}$ concentration in a buffer containing $10 \mathrm{mM}$ potassium phosphate and $100 \mathrm{mM}$ sodium fluoride at $\mathrm{pH}$ 7.4. The protein concentrations were determined using a NanoDropTM One Microvolume UV-Vis spectrophotometer (Thermo Scientific, MA, USA), with theoretical extinction coefficients $\varepsilon_{280}=96,720$ (wild-type MBP-CHIP, G249V, and R51_I53delinsPA) and 91,220 (K143_W147del) $\mathrm{M}^{-1} \mathrm{~cm}^{-1}$. Far-UV spectra were acquired in the range of $185-260 \mathrm{~nm}$ at a scan rate of $50 \mathrm{~nm} / \mathrm{min}$ at $20^{\circ} \mathrm{C}$, using a quartz cell with a path length of $1 \mathrm{~mm}$. Three scans were accumulated for each spectrum and three spectra were buffer subtracted and then averaged. Thermal denaturation profiles were obtained by recording the decrease in ellipticity at $222 \mathrm{~nm}$ as a function of temperature in the range of $20-90{ }^{\circ} \mathrm{C}$ with a scan rate of $40{ }^{\circ} \mathrm{C} / \mathrm{h}$. The results are expressed in mean residue ellipticity $[\theta]_{\mathrm{mrw}}=\theta\left(\mathrm{deg} \times \mathrm{cm}^{2} \times \mathrm{dmol}^{-1}\right)$, and BeSTSel (Beta Structure Selection) was used to estimate the secondary structure content [30]. Final graphs were prepared by using GraphPad Prism software (San Diego, CA, USA).

\subsection{Native Polyacrylamide Gel Electrophoresis (Native-PAGE)}

In total, $5 \mu \mathrm{g}$ of wild-type and mutant MBP-CHIP in Native Sample Buffer (Bio-Rad Laboratories, CA, USA) was loaded in a 10\% Mini-PROTEAN ${ }^{\circledR}$ TGX $^{\mathrm{TM}}$ Precast Protein Gel (Bio-Rad Laboratories) and run at $4{ }^{\circ} \mathrm{C}$ for $3 \mathrm{~h}$ at $140 \mathrm{~V}$ in a Tris/glycine buffer $(25 \mathrm{mM}$ Tris, $192 \mathrm{mM}$ glycine) at $\mathrm{pH} 8.3$ as running buffer. The gel was stained using Coomassie Brilliant Blue G-250.

Supplementary Materials: Supplementary materials can be found at https://www.mdpi.com/ article/10.3390/ijms22115870/s1.

Author Contributions: Conceptualization, S.B., P.M.K., S.J., and I.A.; data curation, Y.P., S.B., H.J.B., and I.A.; formal analysis, Y.P., and H.J.B.; funding acquisition, P.M.K. and S.J.; investigation, Y.P., S.B., H.J.B., and S.E. (Sigrid Erdal); methodology, Y.P. and H.J.B.; project administration, I.A.; resources, B.A.T., P.A.J., K.N.P., M.K., L.I.B., A.S., L.A.B., and P.M.K.; supervision, P.M.K., S.J., and I.A.; validation, Y.P., H.J.B., and I.A.; visualization, Y.P., S.B., H.J.B., S.E. (Sigrid Erdal), K.N.P., A.S., and L.A.B.; writing-original draft, Y.P. and I.A.; writing—review and editing, S.B., H.J.B., B.A.T., P.A.J., K.N.P., S.E. (Ståle Ellingsen), M.K., L.I.B., A.S., L.A.B., P.M.K., and S.J. All authors have read and agreed to the published version of the manuscript. 
Funding: This work was supported by grants from the University of Bergen, Helse Vest's Open Research Grant (grants \#912250 and F-12144), and the Novo Nordisk Foundation (grant NNF19OC0057445).

Institutional Review Board Statement: Not applicable.

Informed Consent Statement: Written informed consent was obtained from all subjects involved in the study.

Data Availability Statement: Data underlying this article can be made available upon request.

Acknowledgments: We wish to thank the patients and their families for participating in this study. We also want to thank Tomasz Stokowy at the Genomics Core Facility, Department of Clinical Science, University of Bergen (Bergen, Norway) for helping to analyze the data retrieved from the whole genome sequencing performed on patient II-1 in Family B.

Conflicts of Interest: The authors declare no conflict of interest.

\section{Appendix A}

A

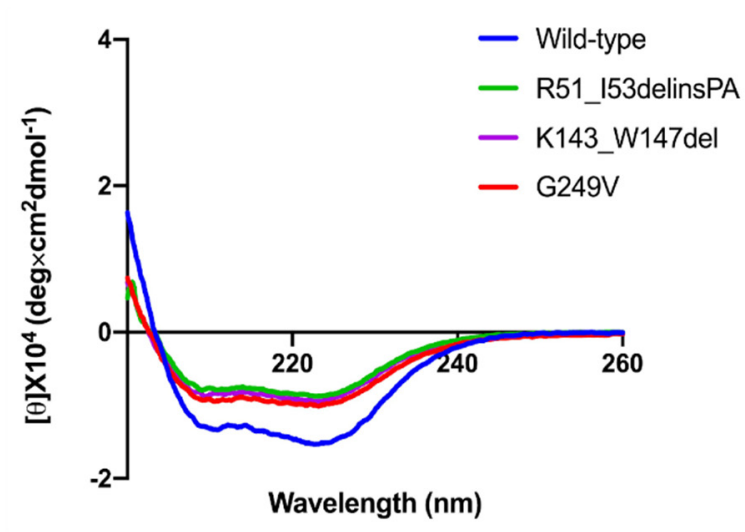

B

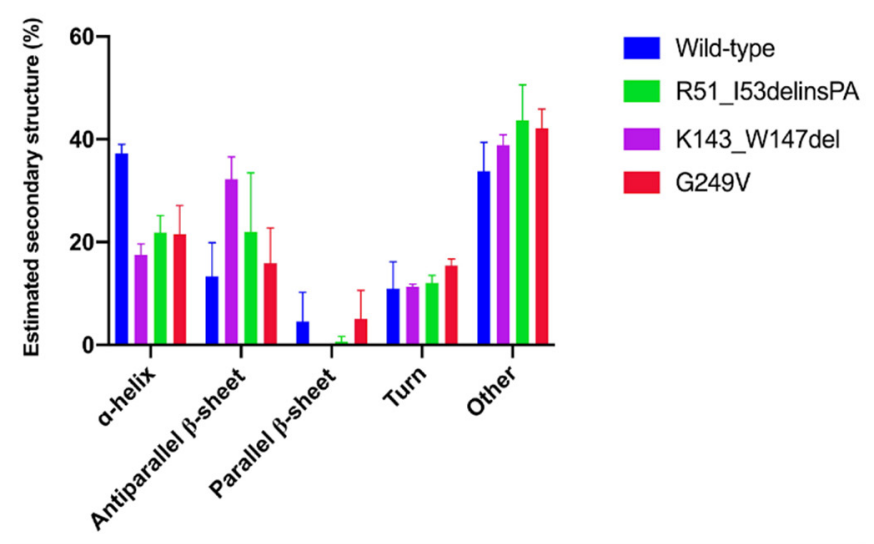

Figure A1. Secondary structure content of wild-type and mutant MBP-CHIP proteins. (A) Far-UV spectra were recorded in the range of 0-260 nm. Final graph was prepared using GraphPad Prism after smoothing. (B) Estimation of the secondary structure content in mutant MBP-CHIP proteins compared to the wild-type, using BeSTSel.

\section{References}

1. Durr, A. Autosomal dominant cerebellar ataxias: Polyglutamine expansions and beyond. Lancet Neurol. $2010,9,885-894$. [CrossRef]

2. Ruano, L.; Melo, C.; Silva, M.C.; Coutinho, P. The global epidemiology of hereditary ataxia and spastic paraplegia: A systematic review of prevalence studies. Neuroepidemiology 2014, 42, 174-183. [CrossRef] [PubMed]

3. Bird, T.D. Hereditary Ataxia Overview. In GeneReviews $\left({ }^{\circledR}\right)$; Adam, M.P., Ardinger, H.H., Pagon, R.A., Wallace, S.E., Bean, L.J.H., Mirzaa, G., Amemiya, A., Eds.; University of Washington: Washington, WA, USA, 1993.

4. Genis, D.; Ortega-Cubero, S.; San Nicolás, H.; Corral, J.; Gardenyes, J.; de Jorge, L.; López, E.; Campos, B.; Lorenzo, E.; Tonda, R.; et al. Heterozygous STUB1 mutation causes familial ataxia with cognitive affective syndrome (SCA48). Neurology 2018, 91, e1988-e1998. [CrossRef] [PubMed]

5. $\quad$ Lieto, M.; Riso, V.; Galatolo, D.; De Michele, G.; Rossi, S.; Barghigiani, M.; Cocozza, S.; Pontillo, G.; Trovato, R.; Saccà, F.; et al. The complex phenotype of spinocerebellar ataxia type 48 in eight unrelated Italian families. Eur. J. Neurol. 2020, 27, 498-505. [CrossRef]

6. De Michele, G.; Lieto, M.; Galatolo, D.; Salvatore, E.; Cocozza, S.; Barghigiani, M.; Tessa, A.; Baldacci, J.; Pappatà, S.; Filla, A.; et al. Spinocerebellar ataxia 48 presenting with ataxia associated with cognitive, psychiatric, and extrapyramidal features: A report of two Italian families. Parkinsonism Relat. Disord. 2019, 65, 91-96. [CrossRef] [PubMed]

7. Chen, D.-H.; Latimer, C.; Yagi, M.; Ndugga-Kabuye, M.K.; Heigham, E.; Jayadev, S.; Meabon, J.S.; Gomez, C.M.; Keene, C.D.; Cook, D.G.; et al. Heterozygous STUB1 missense variants cause ataxia, cognitive decline, and STUB1 mislocalization. Neurol. Genet. 2020, 6, e397-13. [CrossRef]

8. Ravel, J.-M.; Benkirane, M.; Calmels, N.; Marelli, C.; Ory-Magne, F.; Ewenczyk, C.; Halleb, Y.; Tison, F.; Lecocq, C.; Pische, G.; et al. Expanding the clinical spectrum of STIP1 homology and U-box containing protein 1-associated ataxia. J. Neurol. 2021, 268, 1927-1937. [CrossRef] 
9. De Michele, G.; Galatolo, D.; Barghigiani, M.; Iacovo, D.D.; Trovato, R.; Tessa, A.; Salvatore, E.; Filla, A.; De Michele, G.; Santorelli, F.M. Spinocerebellar ataxia type 48: Last but not least. Neurol. Sci. 2020, 41, 2423-2432. [CrossRef] [PubMed]

10. Ballinger, C.A.; Connell, P.; Wu, Y.; Hu, Z.; Thompson, L.J.; Yin, L.-Y.; Patterson, C. Identification of CHIP, a Novel Tetratricopeptide Repeat-Containing Protein That Interacts with Heat Shock Proteins and Negatively Regulates Chaperone Functions. Mol. Cell. Biol. 1999, 19, 4535-4545. [CrossRef]

11. Nikolay, R.; Wiederkehr, T.; Rist, W.; Kramer, G.; Mayer, M.P.; Bukau, B. Dimerization of the Human E3 Ligase CHIP via a Coiled-coil Domain Is Essential for Its Activity*. J. Biol. Chem. 2004, 279, 2673-2678. [CrossRef]

12. Shi, Y.; Wang, J.; Li, J.-D.; Ren, H.; Guan, W.; He, M.; Yan, W.; Zhou, Y.; Hu, Z.; Zhang, J.; et al. Identification of CHIP as a novel causative gene for autosomal recessive cerebellar ataxia. PLoS ONE 2013, 8, e81884. [CrossRef] [PubMed]

13. Pakdaman, Y.; Sanchez-Guixé, M.; Kleppe, R.; Erdal, S.; Bustad, H.J.; Bjørkhaug, L.; Haugarvoll, K.; Tzoulis, C.; Heimdal, K.; Knappskog, P.M.; et al. In vitro characterization of six STUB1 variants in spinocerebellar ataxia 16 reveals altered structural properties for the encoded CHIP proteins. Biosci. Rep. 2017, 37. [CrossRef] [PubMed]

14. Kanack, A.J.; Newsom, O.J.; Scaglione, K.M. Most mutations that cause spinocerebellar ataxia autosomal recessive type 16 (SCAR16) destabilize the protein quality-control E3 ligase CHIP. J. Biol. Chem. 2018, 293, 2735-2743. [CrossRef] [PubMed]

15. Schindelin, J.; Arganda-Carreras, I.; Frise, E.; Kaynig, V.; Longair, M.; Pietzsch, T.; Preibisch, S.; Rueden, C.; Saalfeld, S.; Schmid, B.; et al. Fiji: An open-source platform for biological-image analysis. Nat. Methods 2012, 9, 676-682. [CrossRef] [PubMed]

16. Palvadeau, R.; Kaya-Güleç, Z.E.; Şimşir, G.; Vural, A.; Öztop-Çakmak, Ö.; Genç, G.; Aygün, M.S.; Falay, O.; Başak, A.N.; Ertan, S Cerebellar cognitive-affective syndrome preceding ataxia associated with complex extrapyramidal features in a Turkish SCA48 family. Neurogenetics 2020, 21, 51-58. [CrossRef]

17. Roux, T.; Barbier, M.; Papin, M.; Davoine, C.S.; Sayah, S.; Coarelli, G.; Charles, P.; Marelli, C.; Parodi, L.; Tranchant, C.; et al. Clinical, neuropathological, and genetic characterization of STUB1 variants in cerebellar ataxias: A frequent cause of predominant cognitive impairment. Genet. Med. 2020, 22, 1851-1862. [CrossRef]

18. Park, J.; Deininger, N.; Rautenberg, M.; Saft, C.; Harmuth, F.; Sturm, M.; Riess, O.; Schöls, L.; Synofzik, M.; Haack, T.B. Correspondence on "Clinical, neuropathological, and genetic characterization of STUB1 variants in cerebellar ataxias: A frequent cause of predominant cognitive impairment" by Roux et al. Genet. Med. 2021, 1-2. [CrossRef]

19. Zhang, M.; Windheim, M.; Roe, S.M.; Peggie, M.; Cohen, P.; Prodromou, C.; Pearl, L.H. Chaperoned Ubiquitylation-Crystal Structures of the CHIP U Box E3 Ubiquitin Ligase and a CHIP-Ubc13-Uev1a Complex. Mol. Cell 2005, 20, 525-538. [CrossRef]

20. McLaughlin, B.; Buendia, M.A.; Saborido, T.P.; Palubinsky, A.M.; Stankowski, J.N.; Stanwood, G.D. Haploinsufficiency of the E3 ubiquitin ligase $\mathrm{C}$-terminus of heat shock cognate 70 interacting protein (CHIP) produces specific behavioral impairments. PLoS ONE 2012, 7, e36340. [CrossRef]

21. Shi, C.H.; Schisler, J.C.; Rubel, C.E.; Tan, S.; Song, B.; McDonough, H.; Xu, L.; Portbury, A.L.; Mao, C.Y.; True, C.; et al. Ataxia hypogonadism caused by the loss of ubiquitin ligase activity of the U box protein, C.H.I.P. Hum. Mol. Genet. 2014, 23, 1013-1024. [CrossRef]

22. Heimdal, K.; Sanchez-Guixé, M.; Aukrust, I.; Bollerslev, J.; Bruland, O.; Jablonski, G.E.; Erichsen, A.K.; Gude, E.; A Koht, J.; Erdal, S.; et al. STUB1 mutations in autosomal recessive ataxias-Evidence for mutation-specific clinical heterogeneity. Orphanet $J$. Rare Dis. 2014, 9, 146. [CrossRef]

23. Herrema, H.; Mikkelsen, T.; Robin, A.; LeWitt, P.; Sidiropoulos, C. SCA 17 phenotype with intermediate triplet repeat number. J. Neurol. Sci. 2014, 345, 269-270. [CrossRef] [PubMed]

24. Doherty, K.M.; Warner, T.T.; Lees, A.J. Late onset ataxia: MSA-C or SCA 17? A gene penetrance dilemma. Mov. Disord. 2014, 29, 36-38. [CrossRef] [PubMed]

25. Jih, K.-Y.; Lin, K.-P.; Tsai, P.-C.; Soong, B.-W.; Liao, Y.-C.; Lee, Y.-C. Investigating TBP CAG/CAA trinucleotide repeat expansions in a Taiwanese cohort with ALS. Amyotroph. Lateral Scler. Front. Degener. 2020, 2020, 1-6.

26. Shin, J.H.; Park, H.; Ehm, G.H.; Lee, W.W.; Yun, J.Y.; Kim, Y.E.; Lee, J.-Y.; Kim, H.-J.; Kim, J.-M.; Jeon, B.S.; et al. The Pathogenic Role of Low Range Repeats in SCA17. PLoS ONE 2015, 10, e0135275. [CrossRef] [PubMed]

27. Wu, C.; Cai, Q.; You, H.; Zhou, X.; Chen, D.; Mo, G.; Li, X. Co-occurrence of ATXN3 and ATXN2 repeat expansions in Chinese ataxia patients with slow saccades. Mol. Genet. Genom. Med. 2019, 7, e663. [CrossRef]

28. Holtan, J.P.; Aukrust, I.; Jansson, R.W.; Berland, S.; Bruland, O.; Gjerde, B.L.; Stokowy, T.; Bojovic, O.; Forsaa, V.; Austeng, D.; et al. Clinical features and molecular genetics of patients with ABCA4-retinal dystrophies. Acta Ophthalmol. 2020. [CrossRef]

29. Bredrup, C.; Johansson, S.; Bindoff, L.A.; Sztromwasser, P.; Kråkenes, J.; Mellgren, A.E.; Brurås, K.R.; Lind, O.; Boman, H.; Knappskog, P.M.; et al. High Myopia-Excavated Optic Disc Anomaly Associated With a Frameshift Mutation in the MYC-Binding Protein 2 Gene (MYCBP2). Am. J. Ophthalmol. 2015, 159, 973-979e2. [CrossRef] [PubMed]

30. Micsonai, A.; Wien, F.; Bulyáki, É.; Kun, J.; Moussong, É.; Lee, Y.-H.; Goto, Y.; Réfrégiers, M.; Kardos, J. BeStSel: A web server for accurate protein secondary structure prediction and fold recognition from the circular dichroism spectra. Nucleic Acids Res. 2018, 46, W315-W322. [CrossRef] 